confidence in pain assessment was from 2.32 to 4.02 , complex communication skills from 2.82 to 4.20 , cultural care from 2.53 to 3.75 .

Conclusion Programme has received positive feedback with increase in knowledge, skills and confidence. Attendees are cascading their learning to other healthcare professionals in their clinical areas supported by their mentor. The challenge of some missed session by attendees will be addressed by encouragement. Based on the results, programme for healthcare assistants is being developed.

\section{DEVELOPING THE PALLIATIVE CARE CLINICAL NURSE SPECIALIST WORKFORCE - AN INNOVATIVE APPROACH}

Lesley Charman, Charlotte Rock, Elizabeth Price, Vanessa Taylor, Kathryn Sartain, Catherine Malia. Leeds Teaching Hospitals NHS Trust, Harrogate and District Foundation Trust, University of Huddersfield, York NHS Trust, St Gemma's Hospice

\subsection{6/spcare-2020-PCC.34}

Background The specialist palliative care workforce is experiencing recruitment and retention challenges to Clinical Nurse Specialist (CNS) roles in all settings. In response, drawing on the End of Life Care Learning Outcomes (LO) (Taylor 2016), the Yorkshire CNS Workforce Group created a supra-regional CNS development programme (Dec 2019-July 2020) supported by HEE funding.

Twenty two participants across thirteen sites are supported by a local mentor to:

- develop individual workplace development programmes using the LOs

- receive support/on-going education

- record evidence of achievement in the LO document.

Project aims:

- sharing and delivery of education resources to multiple participants

- formation of a community-of-practice for CNSs'/mentors

- implementation of LOs using bespoke documentation, enabling regional benchmarking of CNS competence

- development of e-portfolio enabling 'pass-porting' between organisations (hosted on HEE Website)

- improve CNS confidence/knowledge/retention

Method Utilising the ECHO tele-education model, 6 interactive education sessions (presentation and case based discussion) will be live-streamed to participants in their place of work. Participants should participate in a minimum of 5 sessions. A participant focus group identified topics, ensuring relevant, practice-related learning. Participants and mentors implement individual workplace development programmes.

Evaluation To evaluate impact on knowledge, confidence, staff retention.

Completion of self-evaluation in relation to Learning Outcomes using Bondy Self-Assessment tool at beginning, during and end of project. Completion of validated Self-efficacy questionnaire pre and post course. Immediate evaluation using ECHO technology after each taught session (Likert scale). Comparative recruitment data analysis at end of project

Results Initial Feedback after first session, all 20 participants agree or strongly agree that:

- Participation increased knowledge and confidence

- They felt connected to other participants
- Topic was relevant to practice and LO

This innovative, collaborative project brings new CNSs together across a region and beyond, learning and developing together in a virtual cohort, to build confidence and knowledge.

\section{CLINICAL INDICATION DOCUMENTATION WITH DEXAMETHASONE: AN AUDIT OF INDICATION DOCUMENTATION IN PATIENTS TREATED WITH DEXAMETHASONE IN AN IN-PATIENT HOSPICE UNIT}

Niamh M Cleary. Our Lady's Hospice and Care Services, Dublin

\subsection{6/spcare-2020-PCC.35}

Background Dexamethasone is a corticosteroid with potent glucocorticoid but limited mineralocorticoid activity suitable for high dose anti-inflammatory therapy.It is used in Palliative Medicine for a variety of licenced and unlicenced indications. Glucocorticoid toxicity depends on the dose that is administered over a certain period of time. Therefore even low doses can have toxic effects if administered long-term. Because dexamethasone is used for various indications in palliative care it is pertinent that the indication is clearly documented in order to facilitate medication review. The aim of this audit is to identify those patients treated with dexamethasone on a single day in an in-patient hospice unit and to identify if indication for treatment with dexamethasone has been documented

Methods A manual review of patient medication records was carried out on a single day in a 36- bed in-patient hospice unit to identify patients prescribed dexamethasone. Documentation of indication in either the patient medication record or patient clinical record was audited. Education was provided to medical staff. Subsequent re-audit took place to complete the audit cycle.

Results Initial data collection identified 8 patients on current dexamethasone treatment. 2 had clinical indication documented. The range prescribed was $2 \mathrm{mg}-6 \mathrm{mg}$. Mean prescribed was $3.625 \mathrm{mg}$ and median was $4 \mathrm{mg}$.

Following re-audit, 10 patients were identified on current dexamethasone treatment. 5 had clinical indication documented and five did not. The range of dexamethasone prescribed was 1-24 mg. The mean prescribed was $5.1 \mathrm{mg}$ and median was $2 \mathrm{mg}$.

Conclusions Dexamethasone is used for both licenced and unlicenced indications in the palliative care setting. Because its toxicity is dependent on the dose that is administered over a certain period of time, the smallest dose for the shortest period of time is crucial in limiting toxicity. This audit has shown that a brief educational session improves documentation of clinical indication for dexamethasone.

\section{HOW SHOULD THE PALLIATIVE MEDICINE ASPECT OF THE NEW INTERNAL MEDICINE CURRICULUM BE BEST IMPLEMENTED?}

Alice Crabtree, Annell Prosser, Fiona Rawlinson. NHS

10.1136/spcare-2020-PCC.36

Background With the introduction of phase 1 of the new Internal Medicine curriculum in August 2019, there is a need 